\title{
A TEM Study of Wet Oxidation of InAIP on GaAs
}

\author{
J. Zhang*, Y, Cao*, T.H. Kosel*, R.E. Cook** and D.C. Hall* \\ * Dept. of Electrical Engineering, Univ. of Notre Dame, Notre Dame, IN 46556 \\ ** Electron Microscopy Center, Argonne National Laboratory, Argonne, IL 60439
}

Wet oxidation of an MOCVD film of InAlP on GaAs produces an oxide film with excellent electrical properties. Recent work [1 ] has demonstrated successful GaAs MOSFETs using this oxide as the gate dielectric.

The InAlP thin films were grown on GaAs by MOCVD, and later oxidized at $500{ }^{\circ} \mathrm{C}$ in $\mathrm{H}_{2} \mathrm{O}$ saturated $\mathrm{N}_{2}$. TEM cross sections were prepared by wedge polishing, and $2 \mathrm{kV}$ Ar ion milling. The oxide is generally amorphous, showing no sharp diffraction rings, but HRTEM images also show some light nanocrystalline particles in the bulk of the oxide.

Dark particles $\sim 10 \mathrm{~nm}$ in diameter are observed at the bottom of the oxide in BF images of both fully and partially oxidized InAlP films, and are believed to be detrimental to electrical properties.

Thinner oxides, produced by shorter oxidation times or full oxidation of thinner InAlP films, contain smaller particles (Fig. 1). In the thinnest oxides ( $\leq 17 \mathrm{~nm})$, no particles were observed at all [2].

The fact that the particles are dark in BF images suggests that they have higher average atomic number $(Z)$ than the surrounding oxide, giving dark mass-thickness contrast. EDXS spectra cannot be used to determine the particle composition, because focusing the electron beam to a sufficiently small $(10 \mathrm{~nm})$ probe severely damages the film, producing a light area surrounded by newly formed dark particles. In addition, particles in cross sectional specimens disappear rapidly during HREM imaging, evidently by evaporation into the TEM vacuum. However, they are stable in plan-view specimens (Fig. 3), because they are separated from the vacuum by the semiconductor on one side and the oxide on the other. STEM imaging of the particles is possible, due to the short dwell time of the probe on any point of the image. The fact that the particles are lighter than the surrounding oxide in the Z-contrast STEM image in Fig. 2b demonstrates that they have higher average Z, and we conclude that they must be In-rich. This is consistent with Auger depth profiling results obtained by Graham et al. [3 ]. Rapid multiple-scan EDXS line profiles across particles in cross-sectional specimens initially show more In counts at the particle location, but only for the first few scans, because of increasing beam damage to the oxide and the dark particles.

To test the hypothesis that the dark particles form due to slower diffusion of In to the outer surface of the oxide, a diffusion marker experiment was done by wet oxidation of InAlP with small $\mathrm{Cr}$ particles on the surface. Since the Cr markers were found on the outer surface of the oxide (Fig. 4), oxidation must occur by inwards diffusion of oxygen to the semiconductor surface, rather than outwards diffusion of $\mathrm{In}, \mathrm{Al}$ and $\mathrm{P}$. A possible explanation is that rather than being formed during oxidation, the particles may form during cooling, due to decreasing solubility. A diffusion gradient in the oxide could explain their proximity to the interface [4]. 
References

[1] X. Li et al., IEEE Elect. Dev. Lett. 25 (2004) 772-774.

[2] Y. Cao et al., Appl. Phys. Lett. 86, 062105 (2005).

[3] M.J. Graham et al., J. Corrosion Science and Engr. 6 (2003) paper H015.

[4] This research was supported by AFOSR grant AF-F49620-01-1-0331, and U.S. DOE contract W-31-109-Eng-38 (for work done at Argonne National Laboratory).
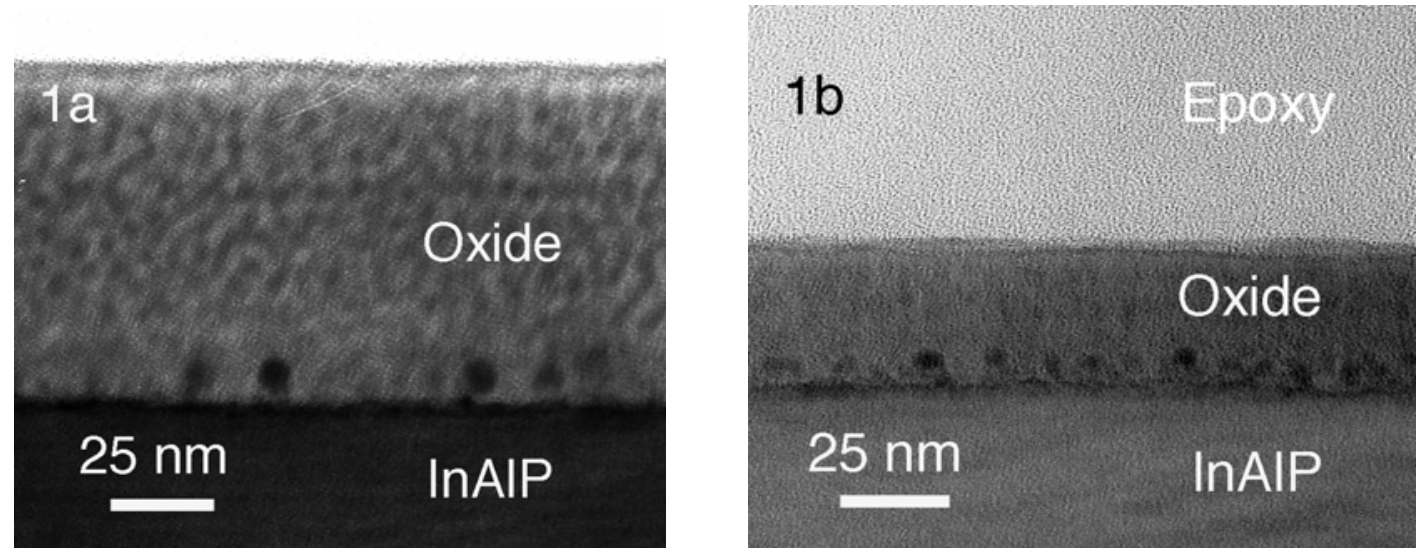

Fig. 1. BF TEM images of oxide formed by a) 60 , and b) 15 min. wet oxidation.
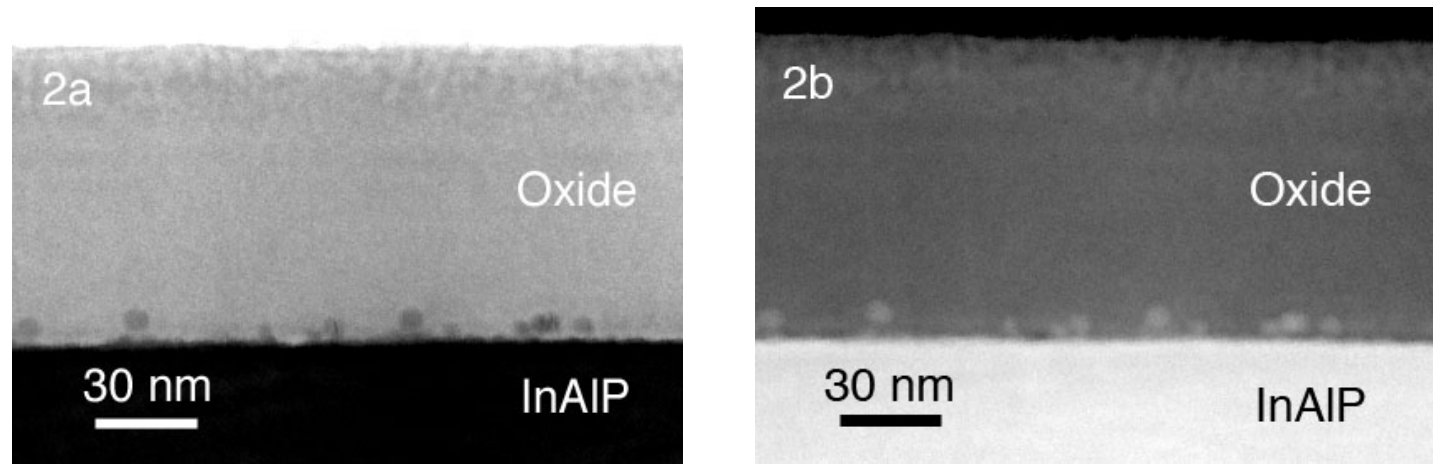

Fig. 2. a) BF, and b) Z-contrast STEM images of partially oxidized thick InAlP.

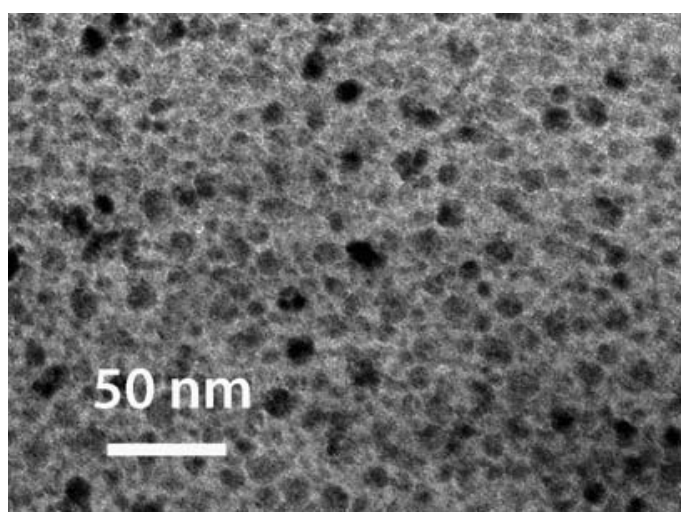

Fig. 3. Plan-view BF image.

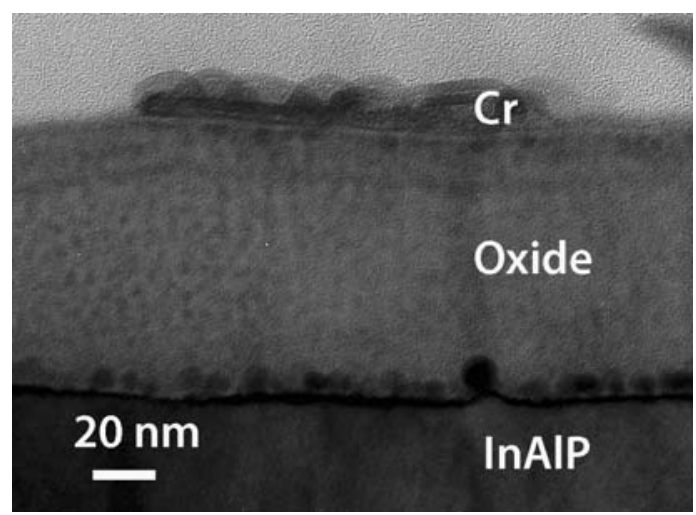

Fig. 4. Cr marker at top of oxide. 